\title{
Dental management in patients with hemostasis alteration
}

\author{
Ariadna Claramunt Lozano ${ }^{1}, \mathbf{M}^{\mathrm{a}}$ Gracia Sarrión Perez ${ }^{2}$, Carmen Gavaldá Esteve ${ }^{2}$ \\ ${ }^{1}$ Degree in Dentistry. Master in Oral Medicine and Surgery. Faculty of Medicine and Dentistry. University of Valencia. Spain. \\ ${ }^{2}$ Valencia University, Department of Stomatology, University General Hospital, Valencia, Spain.
}

Correspondence:

C/Serpis, 66-81. CP 46022

Valencia.

E-mail: ariclaramunt@hotmail.com

Claramunt Lozano A, Sarrión Perez MG, Gavaldá Esteve C. Dental management in patients with hemostasis alteration. J Clin Exp Dent. 2011;3(2):e120-6.

http:/www.medicinaoral.com/odo/volumenes/v3i2/jcedv3i2p120.pdf

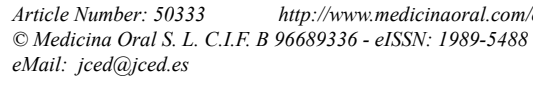




\section{Introduction}

A common complication that arises in patients with disorders of hemostasis after oral surgery is excessive bleeding. The best strategy to avoid these hemorrhagic complications in interventions in the oral cavity is prevention. This approach finds indispensable a detailed medical history of the patient (1-4) and requires comprehension of the physiology of haemostasis. The medical history will emphasise on previous bleeding events after any intervention, labor or dental extraction, as well as any personal and family precedents that could influence an effective hemostasis. Figure 1 shows the current coagulation cascade.

Coagulopathies constitute a heterogeneous group of diseases that cause hemorrhagic diatheses, and are consequence of quantitative or qualitative alterations of the plasma proteins involved in any of the phases of blood coagulation (platelet, vascular, coagulation, fibrinolysis). Diseases are classified into congenital or inherited and acquired, being possible to have both types coexisting in the same patient, as for example a patient with haemophilia A and liver disease. Von Willebrand disease (vWD) is the most common within these inherited diseases, caused by deficiency in quantity or fault in functionality of the Von Willebrand factor (vWF). VWD transmits commonly as autosomal dominant or less frequently as recessive, with an estimation between $1 \%$ and $2 \%$ of affected population. Moreover, hemophilia is also an inherited disease consequence of the activity deficiency of Factor VIII (FVIII) (Hemophilia A or classic) or Factor IX (FIX) (Hemophilia B or Christmas disease). The prevalence is estimated of 1-5.000 in males with hemophilia A and of 1/30.000 with hemophilia B. Hemophilia is transmitted as recessive inheritance associated to chromosome $\mathrm{X}$ (heritage linked to gender), and consequently women pass on the defect without suffering the effects.

Antiplatelet drugs are frequently used for prevention of arterial and venous thrombosis in patients suffering diseases like ischemic cardiopathy, as well as on carriers of prosthetic heart valves and coronary endoprosthesis (stent) and patients with risk of experimenting an ischemic stroke. Furthermore, patients following treatments with antiplatelet drugs have an increased tendency to general bleeding, as antiplatelet mechanism consist in the inhibition of platelet functions. Dentistry professionals
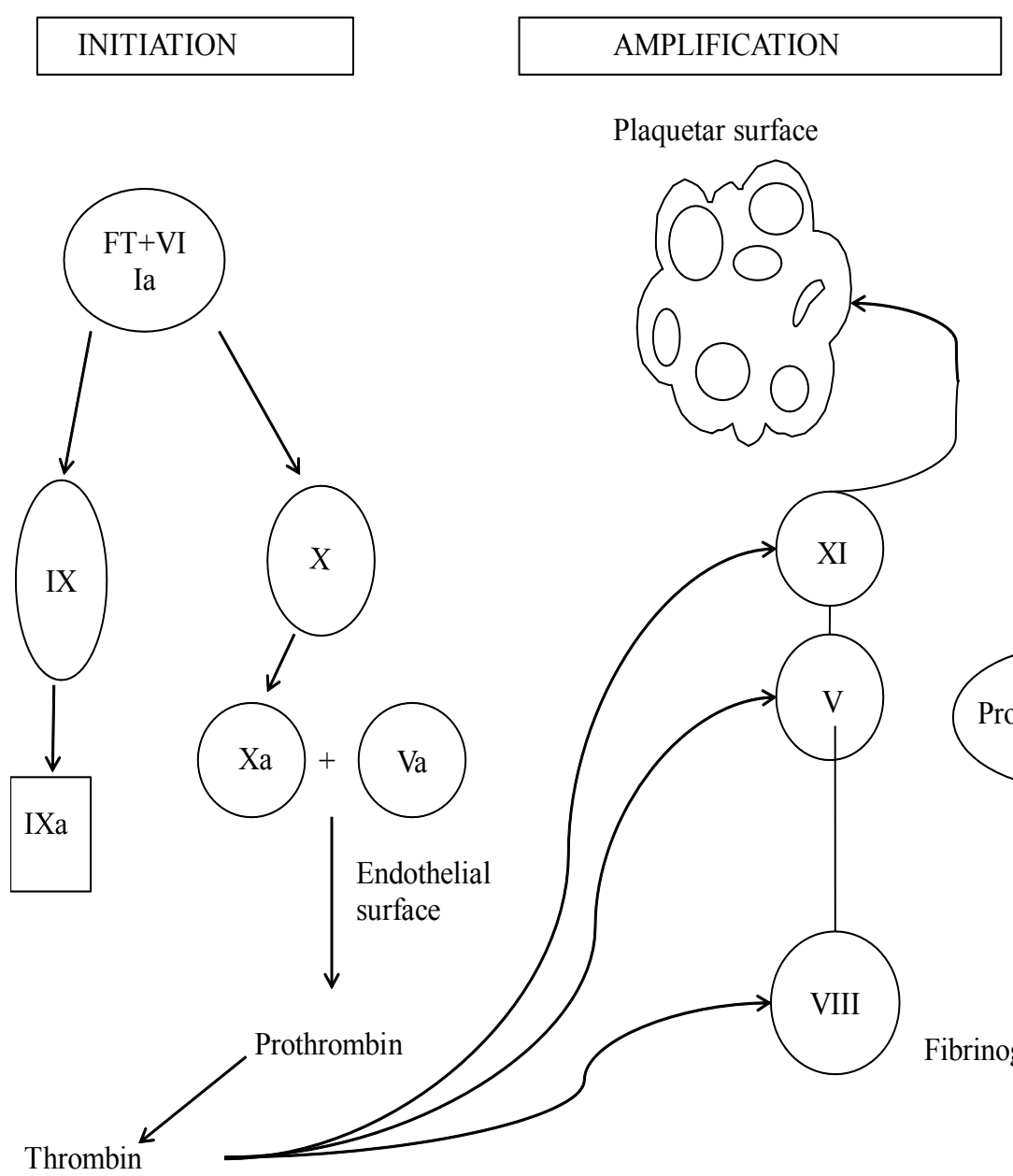

Plaquetar surface

Fig. 1. Current coagulation cascade. 
have to take into account the risk of an oral hemorrhage during and after an invasive dental procedure. Patient's carriers of coronary stents are subject to single or combined antiplatelet therapy. On the other hand, oral anticoagulants are used in treatments for venous thromboembolism and in the prevention of heart attack and systematic embolism in patients with auricular fibrillation, cardiac valvular disease or with metallic valve prostheses. Now a days, warfarin sodium and acenocoumarol are the most common oral anticoagulants; these drugs require a correct monitorisation with INR (International Normalized Ratio) (5) in order to adjust the dose and achieve the desired therapeutic action. The objective is to minimise any adverse effects consequence of either the excess of anticoagulation producing hemorrhages or not reaching the required level leading to thrombosis. This is critical when these patients are subject of surgical procedures like tooth extractions, and there are established guidelines to proceed.

In general terms, almost all authors agree that stopping anticoagulant or antiplatelet therapy is dangerous. This will increase blood clotting capacity or platelet aggregation, rising the risk of thromboembolism episodes.

The objectives of this paper are:

- Carry out a literature review of the pathological conditions that can produce disturbances in the coagulation or platelets aggregation, including both inherited as well as acquired.

- Establish the guidelines in dental management of the patients with disorders in blood coagulation and platelet aggregation.

\section{Material and Methods}

The methodology used through the research for the literature review includes de following sources: PubmedMedline database, the medical journal Medicine, the data base of University of Valencia and specialised published books in general medicine as well as in dentistry.

In Pubmed-Medline data base, the research proceeded by searching the next keywords: hemostasis alteration, hemostasis disorder, blood coagulation disorders, dental management anticoagulated, oral surgery anticoagulated, antithrombotic drugs, tranexamic acid, epsilonaminocaproic acid, INR, warfarin, aspirin.

In order to narrow down the research, the data was filtered down limiting to papers in English and Spanish, within dental journals and not older than 10 years.

\section{Results and Discussion}

\section{Congenital bleeding disorders:}

- Von Willebrand disease

The vWD is the most frequent inherited pathology, caused by the deficiency in quantity or fault in functionality of the vWF (4,6-11), and as mentioned its transmission is autosomal dominant or less frequently as recessive. It is estimated that $1 \%$ to $2 \%$ of the population is affected by the vWD (12).

The $\mathrm{vWF}$ is a synthetic glycoprotein synthesized and stored in the megakaryocyte and in the endothelial cells, which function is to act as a bridge between platelets and sub endothelial structures, amid them, collagen benefits the platelets adhesion. Three main functions are identified: platelets adhesion to the endothelium, adhesion between platelets and the transportation of FVIII $(4,6)$. VWD constitute an heterogeneous group with a large phenotypic variety, which justifies the different grades of haemorrhage intensities, predominantly mild variety. The vWD can be classified into different types depending on the amount of vWF affected $(6,10)$ :

- Type I is the most common, it is autosomal dominant $(70-75 \%)$; consists of a quantitative decrease of $\mathrm{vWF}$ in the plasma.

- $\quad$ Type II, the vWF is qualitatively abnormal, deriving into anomalous binding to its platelet receptor, the Glycoprotein Ib (GPIb); identifying within it three subtypes.

- Type III is a severe variant, transmits as an autosomal recessive inheritance making patients incapable of synthesising vWF $(4,6)$.

The main symptoms may arise as mucosal hemorrhage (menorrhagias, epistaxis,...) (6,9-11), bleeding after dental extraction or postpartum (1-4).

The therapeutic options for the vWD are: 1-Deamino8-D-arginine Vasopressin (DDAVP), antifibrinolytic agents, sinthetic estrogen and substitutive treatments with FVIII concentrate rich in vWF $(1,4,6)$.

Depending on the cuantity and type of hemorrhage as well as of the reaction obtained from the DDAVP, they can be administrated alone or combined.

The DDAVP (Desmopressin Acetate) is a synthetic analogous of the vasopressin capable of releasing from the vascular vWF endothelial to the bloodstream, increasing 4-5 times its basal value during 8-10 hours. The effectiveness is subjected to patients that synthesize normally the vWF, with type I (10). The dose for the intravenous injection is of $0,3 \mu \mathrm{g} / \mathrm{kg}$ in $50-100 \mathrm{cc}$ salino during a period of time of 20-30 minutes. It can also be administrated through subcutaneous injection or intranasal (1). The substitutive treatment with hemoderivatives is effective in all subtypes of the vWD, however, due to the residual risk of transmissible diseases and its high cost, this are held for patients that are not eligible for the farmacologic treatment (without response to the DDAVP, Tachyphylaxis, etc.)

- Hemophilia A and B:

First known congenital coagulopathy and the most common. Is an inherited disease consequence of the lack of activity of the FVIII (hemophilia A or classic) or the FIX (hemophilia B or Christmas disease) (5). The prevalence is estimated to be of 1-5.000 males in haemophilia A and 
$1 / 30.000$ in type B $(1,4)$. The transmission is as a recessive inheritance associated to chromosome $\mathrm{X}$ (heritage linked to gender), resulting in female transmitting but not suffering it, except for the infrequent case where father is hemophilic and the mother is a carrier $(1,4,6,12)$. Both haemophilias, A and B are clinically indistinguishable.

Hemophilia can be classified as severe, moderate and mild. Severe haemophilia will normally be diagnosed before six months and its main characteristics are an activity of the FVIII below $1 \%$ of the normal activity and spontaneous bleeding. Moderate will show activity values between $1 \%$ and $5 \%$ and with hemorrhage episodes after surgery, small traumatisms and it arises around 2 years. The classified as mild has an activity of $5 \%-25 \%$ of the normal activity and haemorrhage will occur after major surgery or intense traumatism.

The number of the hemorrhagic episodes will be different in each patient. The most frequent is in the locomotor system, in particular the articular bleeding or hemarthrosis, arising in the early childhood $(1,4,6,12)$.

The moderate or mild A type hemophiliacs can be treated in most occasions with DDAVP and antifibrinolytic agents. In those classified as severe, it is mandatory the use of substitutive treatments with FVIII or FIX, as required, which can be as purified or combinant plasma concentrate $(1,4,6)$. The dosage will be the same as for the vWD and it is necessary reaction test to DDAVP in patients with type A moderate or mild, as it may allow avoiding the administration of concentrates.

- Dental considerations in the patient with vWD and hemophilia

In patients with moderate or mild hemophilia, the nonsurgical dental practises may proceed under the administration of antyfibtinolytic agents (tranexamic acid or epsilon-aminocaproic acid) and consulting with a haematologist before making any other procedure.

In patients with mild hemophilia A (FVIII $>10 \%$ ) and the majority of patients with vWD (type I), it is possible to dental prophylaxis and some minor surgical intervention under administration of DDAVP.

In patients with severe hemophilia, it is necessary the replacement of the factor before the surgery, local anesthetic injections or dental prophylaxis. As for example, with a patient with type A hemophilia, before a dental extraction it is recommended a dose of $50 \mathrm{UI} / \mathrm{kg}$ of FVIII; and in the case it is type B, the dose will be of $100 \mathrm{IU} / \mathrm{kg}$ of FIX.

Previous to dental extractions it can also be administrated epsilon-aminocaproic acid (Caproamin $\left.{ }^{\circledR}\right)$ or tranexámic acid (amchafibrin ${ }^{\circledR}$ ), at prophylactic doses of $20 \mathrm{mg} / \mathrm{kg}$ every 9 hours, starting 48 hours before the intervention and continuing until the surgical wound closes (7-10 days after) $(4,12)$.

Economic and safety method to control the hemorrhage, is to rinse the mouth before and after the dental extraction with tranexamic acid. The recommendation is to rinse with $10 \mathrm{ml}$ of a $5 \%$ solution during two minutes, four times a day during 7 days. Additionally, before any invasive procedure, including dental manipulation, it is necessary the detection of any FVIII inhibitors.

Patients with antiplatelet drugs

- Antiplatelet agents:

Antiplatelet drugs are frequently used for prevention of arterial and venous thrombosis in patients suffering ischemic cardiopathy, as well as on carriers of prosthetic heart valves and coronary endoprosthesis (stent) and patients with risk of experimenting an ischemic stroke. This treatment provides an evident benefit to patients that has suffered, recent or non-recent, acute myocardial infarction (AMI), cerebral straction, transitory cerebral ischemia and patients classified as high risk for thrombotic episodes (13). The most used drug is Acetylsalicylic acid, with clopidogrel and dipiridamol (14) and the trifusal.

- Acetylsalicylic acid (ASA) (Aspirina ${ }^{\circledR}$, Adiro ${ }^{\circledR}$, Biopak ${ }^{\circledR}$, Tromalyt $\left.{ }^{\circledR}\right)$ :

ASA is the only non-steroidal anti-inflammatory medicament used in the prevention and treatement of thromboembolic disease $(14,15)$. The ASA de-activates the Cyclooxygenase enzyme, making it irreversible for platelet life cycle (15). This enzyme is responsible for the development of prostaglandins and thromboxane A2, involved in the activation mechanisms and platelet aggregation. This defect will persist during platelet lifetime, 10 days (14). The range of dose is between $20-300 \mathrm{mg} /$ day.

Several studies conclude, that in order to prevent, in the long term, thrombotic events in high-risk patients, it is recommended the daily use of AAS. A daily dose of AAS of 75-150 mg will help prevention without any significant increase of bleeding during and after dental extraction $(14,15)$.

- $\quad$ Clopidogrel (Plavix $\left.{ }^{\circledR}\right)$ y Ticlopidine (Tiklid $\left.{ }^{\circledR}\right)$ : Clopidogrel and Ticlopidine are members of the family of the thienopyridines. This antiplatelet drugs act over the receptors of the adenosine diphosphate, involved in platelets aggregation. They are recommended in resistant thromboembolic diseases or intolerant to ASA, as well as if there is a higher risk of cerebral ischemia.

The Ticlopidine (Tiklid $\AA$, Ticlodone ${ }^{\circledR}$ ), seems to be more effective than ASA for the prevention of cerebrovascular accidents however it has more side effects, like anaemia and neutropenia. The diary dose is of $250 \mathrm{mg} /$ day.

- Dipyridamole (Persantin $\left.{ }^{\circledR}\right)$ :

Dipyridamole action mechanism is the inhibition of platelets aggregation increasing the levels of 3 ' 5 '-adenosine cyclic monophosphate. In addition it will also have vasodilating effects due to the stimulus it exerts over the 
synthesis of prostacyclin. On its own it has no influence on the hemorrhage time nor in the platelets aggregation. The side effects are headache, dizziness, muscle weakness and gastrointestinal symptoms. The dosage is 75 $\mathrm{mg} /$ day.

\section{- Triflusal (Disgren):}

In this case, Triflusal acts inhibiting the synthesis of the prostanoids through an irreversible blockage (by acetylation) of cyclooxygenase, impeding de physiological synthesis of antiplatelet factors (prostacycline) and proagregants (thromboxane A2). The recommendation on the dosage is in adults of $300 \mathrm{mg} / 24 \mathrm{~h}$.

- Dental management of patient with antiplatelet drugs: A few years ago, due to the haemorrhage risk in the postsurgery, the recommendation was to withdraw antiplatelet use from 7 to 10 days before the intervention. Now a day, even though every dental invasive procedure has associated a risk of oral hemorrhage, it is not specified to stop the antiplatelet treatment, as the risk of thromboembolism associated could be higher than the risk of haemorrhage.

Therefore, there will not be suppression of the antiplatelet treatment, and the approach will include local measures to ensure a good haemostasis through pressure with a gauze, oxidized celullose, stitches, gauze soaked with tranexamic acid (Amchafibrin $\left.{ }^{\circledR}\right)(13,15)$.

- Coronary Stents:

The stent is a mechanical device that holds the artery open, with a structure obtaining a more circular circumference, and smooths the internal contour of the artery. The material has to be biocompatible and resistant to thrombosis, to migracion and to the extrinsic compression (radial force).

Stents are classified into two different groups, metallic and pharmaco-active (the drug is incorporated in a polymeric mold attached to the metallic surface of the stent, and will release the medicine in controlled basis to the arterial wall).

Dental considerations of the patient with coronary stents:

Patients that carry stents, metallic or pharmaco-active stents will both receive a treatment with aspirin, usually combined with clopidogrel (has been proved to be more effective in combination, to reduce the risk of cardiac episodes). The guidelines for patients carriers of stent are the following (16):

- Minor surgery, dental prophylaxis or dental extractions can be performed with a small risk of hemorrhage, without the need of withdrawing the antiplatelet treatment (using local measures to ensure a good hemostasis) (13).

- Non-urgent invasive procedures with significant risk of hemorrhage will be delayed at least one month for patients with metallic stents and to 12 months to patients with pharmaco-active stents, instead of withdrawing the antiplatelet activity.

- In patients that it is necessary a surgical intervention that requires the withdraw of antiplatelet treatment with clopidogrel, the aspirin will not be withdrawn and clopidogrel will be re-established as soon as possible to prevent thrombosis of the stent.

Anticoagulated patients:

- Oral anticoagulants (OAC):

$\mathrm{OAC}$ are used in treatments for venous thromboembolism and in the prevention of heart attack and systematic embolism in patients with auricular fibrillation, cardiac valvular disease or with metallic valve prostheses $(3,17$ $19)$. Now a days, warfarin sodium (Coumadin $\left.{ }^{\circledR}\right)(14,17$ 19), is the most popular in Anglo-Saxon countries and acenocumarol (Sintrom $\AA$ ) is the most used in Spain, which is a derivative of the 4-hydroxycoumarin. Is an antagonist of the $\mathrm{K}$ vitamin, and inhibits the carboxylation of glutamate in the amino terms of these proteins. The warfarin has two functions: anticoagulant activity and antithrombotic effect. The ACO is monitored by its effect on the Prothrombin time (PT). Generally, the PT results will be expressed as time of patient / time of control. In 1983 the WHO introduced the INR (International Normalized Ratio). The therapeutic levels of anticoagulation that should be preserved on patients depending on the kind of pathology will oscillate the INR between 2 to 3 (14,17-19), except for cardiac valvular prosthesis that should have INR maintained between 2,5 and 3,5 (1). Existing a strong correlation between INR and risk of hemorrhage, increasing when the INR $>3$.

- Dental management of the anticoagulated patient:

The procedure with a patient with coagulation disturbances that is going to be subject to a dental extraction is described in the following recommended guideline:

1. The INR must be checked the same day of the surgery. A simple exodontia will take place if the results are within the therapeutic values (2-3 or 2,53,5 , depending on the pathology) (Normal values of INR is 1.0). If the results are within these therapeutic values, there will not be any modification in the anticoagulant treatment (14,17-19). Applying local measures can control the possible hemorrhage, and withdrawing the treatment will not necessarily reduce the hemorrhage and could lead to thromboembolic complications.

In the case of a surgical exodontia, it will be required to consult a haematologist, in order to decide on the withdrawal of the ACO 2-3 days before the intervention, substituting it by low molecular weight heparin (LMWH). Be administered prophylactic doses of LMWH if the INR daily checked is $<2.0$, overlapping two anticoagulants from the day after surgery, to achieve the desired values (3).

2. The dental extraction will be done as carefully as possible, minimising traumatism of soft tissue, 
avoiding any major hemorrhage.

3. Once the exodontia is completed, proceed with local hemostatic measures with re-absorbable fibrin adhesives (Tissucol ${ }^{\circledR}$ ), oxidized cellulose or gelatin sponges to help clot formation $(8,17-20)$.

4. Proceed with silk or re-absorbable stitches (in order to reduce the risk of hemorrhage when take off), joining both sides, and impede the release of the hemostatic agent from the alveolus.

5. Compression with a gauze soaked in tranexamic acid (Amchafirin $\AA$ ) $(8,14,17,18)$, during 20-30 min.

6. Revision of the surgical wound after $20 \mathrm{~min}$.

Prevention is the most important measure of them all (8). In table 1, a number of recommendations can be found to follow in patients with hemostasis disturbances.

The main complication that may arise, in patients with hemostasis alterations as well as in anticoagulated and antiagregated patients, is a post-surgical hemorrhage.
The recommended guidelines are described in table 2 .

Conclusively, a number of protocols and guidelines have been defined for the procedures with anticoagulated and antiagregated patients, from the odontological point of view.

In patients with antiplatelet drugs that are subject of a simple therapy there will not be modifications in the antiplatelet treatment, instead local measures will be practised in order to ensure a good hemostasis. In combined therapies it will be possible to proceed in the same way involving minor surgery, dental prophylaxis or dental extraction without modifying the antiplatelet treatment. (local haemostatic measures)

Moreover, in patients that it is necessary a surgical intervention that requires the withdraw of antiplatelet treatment with clopidogrel, the aspirin will not be withdrawn and clopidogrel will be re-established as soon as possible.

\section{Prevention of the complications}

1. Identification of the patient by a detailed medical history: disease antecedents, exploration and laboratory tests for selective indentification $(1,2,8)$.

2. Enhance the oral hygiene to avoid invasive dental treatments

3. Consultation with the hematologyst, to know the kind of congenital disorder, or to learn the extent of the pacient anticoagulation.

4. Do not perform surgery if platelets count is less $\tan 50,000$ platelets.

5. Replacement therapy of coagulation factor déficit if necessary.

6. (hemophilia A and B and vWD)(1,10-12).

7. Value the hospital admission for complex surgery in the case of hemophilia.

8. Do not use ASA or its derivatives for treatment of pain. Paracetamol is a safe alternative.

9. Coumarin interactions with another drugs: antibiotics (amoxicillin and amoxicillin plus clavulanate, ampicillin, azithromycin, erythromycin, rifampicin, penicillin G, cephalosporins, sulfonamides, metronidazole, chloramphenicol), antifungals (azoles and griseofulvin), analgesics (aspirin and other nonsteroidal antiinflammatory drugs; paracetamol in excess can enhance warfarin action), and psychoactive drugs (some antihistamines, diazepam) (1).

10. Consultations in the morning, in order to resolve any complication during the day.

11. Avoid, if posible, nerve block anesthesia.

12. Preferable endodontics or restorative treatment to dental extractions.

Table 1. Recomendations to avoid complications.

\section{Treatment of the complications}

1. Remove stitches.

2. Clean the clot to identify the bledding site.

3. Local anesthesia with epinephrine (lidocaine $2 \%$ with epinephrine $1: 80.000$ to $1: 200.000$ ).

4. Tamponade of alveolus, stitches.

5. Compression with gauze soaked with tranexamic acid (Amchafibrin $\AA$ ).

6. Acetate desmopressin if persistent bleeding.

7. Rinses with antifibrinolytics 4 times a day, 7 days (19).

Table 2. Treatment of the complications in patients with hemostasis alterations. 
In anticouagulated patients, the INR will be measured the same day of the intervention. If INR is higher than normal values or it is a surgical intervention, a haematologist should be consulted in order to decide on the withdraw of the ACO treatment, substituting it with LMWH. Complemented with local hemostatic measures.

\section{References}

1. Jover-Cerveró A, Poveda Roda R, Bagán JV, Jiménez Soriano Y. Dental treatment of patients with coagulation factor alterations: an update. Med Oral Patol Oral Cir Bucal. 2007;12:E380-7.

2. Daniel NG, Goulet J, Bergeron M, Paquin R, Landry PE. Antiplatelet drugs: is there a surgical risk? J Can Dent Assoc. 2002;68:683-7.

3. Heit JA. Perioperative Management of the Chronically Anticoagulated Patient. J Thromb Thrombolysis. 2001;12:81-7.

4. Gómez-Moreno G, Cutando-Soriano A, Arana C, Scully C. Hereditary blood coagulation disorders: management and dental treatment. J Dent Res. 2005;84:978-85.

5. Romney G, Glick M. An updated concept of coagulation with clinical implications. J Am Dent Assoc. 2009;140:567-74.

6. Israels S, Schwetz N, Boyar R, McNicol A. Bleeding disorders: characterization, dental considerations and management. J Can Dent Assoc. 2006;72:827.

7. Kottke-Marchant K, Corcoran G. The laboratory diagnosis of platelet disorders. Arch Pathol Lab Med. 2002;126:133-46.

8. Greaves M, Watson HG. Approach to the diagnosis and management of mild bleeding disorders. J Thromb Haemost. 2007;5:16774.

9. Morimoto Y, Yoshioka A, Sugimoto M, Imai Y, Kirita T. Haemostatic management of intraoral bleeding in patients with von Willebrand disease. Oral Dis. 2005; 11: 243-8.

10. Federici AB. Clinical Diagnosis of von Willebrand disease. Haemophilia. 2004;10:169 76.

11. Mannucci PM. Treatment of von Willebrand's disease. N Engl J Med. 2004;351:683-94.

12. Brown DL. Congenital Bleeding disorders. Curr Probl Pediatr Adolesc Health Care.2005;35:38-62.

13. Napeñas JJ, Hong CH, Brennan MT, Furney SL, Fox PC, Lockhart PB. The frequency of bleeding complications after invasive dental treatment in patients receiving single and dual antiplatelet therapy. J Am Dent Assoc. 2009 ;140:690-5.

14. Pototski M, Amenábar JM. Dental management of patients receiving anticoagulation or antiplatelet treatment. J Oral Sci. 2007; 49: 253-8.

15. Brennan MT, Valerin MA, Noll JL, Napeñas JJ, Kent ML, Fox PC, et al. Aspirin use and Post-operative Bledding fron Dental Extractions. J Dent Res. 2008;87:740-4.

16. Grines CL, Bonow RO, Casey DE Jr, Gardner TJ, Lockhart PB, Moliterno DJ, et al; American Heart Association; American College of Cardiology; Society for Cardiovascular Angiography and Interventions; American College of Surgeons; American Dental Association; American College of Physicians. Prevention of premature discontinuation of dual antiplatelet therapy in patients with coronary artery stents: a science advisory from the American Heart Association, American College of Cardiology, Society for Cardiovascular Angiography and Interventions, American College of Surgeons, and American Dental Association, with representation from the American College of Physicians. J Am Dent Assoc. 2007;138:652-5.

17. Vicente Barrero M, Knezevic M, Tapia Martín M, Viejo Llorente A, Orengo Valverde JC, García Jiménez F, et al. Oral surgery in patients undergoing oral anticoagulant therapy. Med Oral. 2002 Jan-Feb; 7:63-6, 67-70.

18. Aframian DJ, Lalla RV, Peterson DE. Management of dental patients taking common hemostasis-altering medications. Oral Surg Oral Med Oral Pathol Oral Radiol Endod. 2007;103:1-11.
19. Jiménez Y, Poveda R, Gavaldá C, Margaix M, Sarrión G. An update on the management of anticoagulated patients programmed for dental extractions and surgery. Med Oral Patol Oral Cir Bucal. 2008;13:E176-9.

20. Sacco R, Sacco M, Carpenedo M, Mannucci PM. Oral surgery in patients on oral anticoagulant therapy: a randomized comparison of different intensity targets. Oral Surg Oral Med Oral Pathol Oral Radiol Endod. 2007;104:18-21. 